\title{
Reduction in miRNA-125b-5p levels is associated with obstructive renal injury
}

\author{
SILU WANG ${ }^{1}$, LIANFENG WU ${ }^{2}$, LINYONG DU ${ }^{3}$, HONG LU $^{2}$, BICHENG CHEN $^{1}$ and YONGHENG BAI ${ }^{1}$ \\ ${ }^{1}$ Key Laboratory of Surgery; ${ }^{2}$ Department of Laboratory Medicine, \\ The First Affiliated Hospital of Wenzhou Medical University, Wenzhou, Zhejiang 325000; \\ ${ }^{3}$ School of Laboratory Medicine and Life Science, Wenzhou Medical University, Wenzhou, Zhejiang 325035, P.R. China
}

Received January 17, 2017; Accepted February 22, 2017

DOI: $10.3892 /$ br.2017.875

\begin{abstract}
Obstructive renal injury is a common disease that leads to progressive glomerulosclerosis, tubulointerstitial fibrosis and loss of renal function. MicroRNAs (miRNAs/miRs) are small non-coding molecules that may be involved in the progression of many renal diseases. The aim of the present study was to investigate the roles of miRNAs, including miR-125b, miR-326 and miR-324p, in obstructive renal injury. Blood samples were collected from 91 patients with ureteral obstruction and 76 controls to examine renal function. In addition, the levels of miR-125b, miR-326 and miR-324p in patients with ureteral obstruction and controls were determined by the reverse transcription-quantitative polymerase chain reaction. Furthermore, the relationship between miRNA levels and renal function was evaluated by the Mann-Whitney U test. Upregulated levels of serum creatinine ( $\mathrm{SCr}$ ) in patients with ureteral obstruction were observed, identifying the injury of renal function. Although the expression levels of miR-324-5p [1.003 (0.391-2.279) vs. $0.934(0.579-1.539), \mathrm{P}=0.300]$ and miR-326 [0.840 (0.180-2.020) vs. 0.949 (0.507-1.702), $\mathrm{P}=0.050]$ presented no significant difference, the levels of miR-125b-5p [0.755 (0.210-2.110) vs. $0.960(0.390-1.770), \mathrm{P}=0.002]$ in patients with ureteral obstruction were significantly lower than those in controls. These results indicated that there is a stronger correlation of miR-125b-5p with the occurrence of ureteral obstruction, especially for the female $(\mathrm{P}=0.0171)$ and elderly $(\mathrm{P}=0.0142)$. Furthermore, the levels of miR-125b-5p $(r=-0.175$, $\mathrm{P}=0.038$ ) were closely associated with the serum levels of $\mathrm{SCr}$, suggesting a key role of miR-125b-5p in renal dysfunction. Thus, these findings suggested that miR-125b-5p in patients
\end{abstract}

Correspondence to: Dr Bicheng Chen or Dr Yongheng Bai, Key Laboratory of Surgery, The First Affiliated Hospital of Wenzhou Medical University, 2 Fuxue Lane, Wenzhou, Zhejiang 325000, P.R. China

E-mail: bisonch@163.com

E-mail: baiyongheng@hotmail.com

Key words: microRNAs, renal function, obstructive renal injury, association with ureteral obstruction correlated with renal function, and may be a potential biomarker for obstructive renal injury.

\section{Introduction}

Ureteral obstruction is a disease that may result from a diversity of congenital or acquired conditions, ranging from calculi to strictures and to inflammatory processes and malignancies (1). The treatment for choice of urinary tract obstruction is to relieve the obstruction. However, sustained ureteral obstruction can eventually lead to progressive glomerulosclerosis, tubulointerstitial fibrosis and the loss of renal function (2-4). Therefore, rapid diagnosis and initiation of the treatment are vital to preserve function and/or to slow down obstructive renal injury.

MicroRNAs (miRNAs/miRs) are endogenous single-stranded small noncoding RNAs of 21-25 nucleotides that regulate gene expression at the posttranscriptional level (5). miRNAs are involved in various cellular biological functions, including apoptosis, proliferation, differentiation and metabolism. The abnormal expression of miRNAs has been reported to be associated with the development of many human diseases (6-8). For example, miR-29a, miR-101-3p and miR-127a are highly expressed in the kidney, where it acts against fibrosis by suppressing collagen expression in tubular cells (9-11). Downregulated serum levels of miR-29a, miR-101-3p and miR-127a have been indicated to predict the degree of acute kidney injury (AKI) in ICU patients (9). Thus, miRNAs may serve important roles in various kidney diseases, and some of them even can be used as biomarkers in early diagnosis and detecting disease development $(12,13)$.

Previous studies of the authors have demonstrated that the activation of the hedgehog signaling pathway contributes to renal injury and fibrogenesis via epithelial-mesenchymal transitions (EMT) in obstructive renal injury $(14,15)$. Inhibiting the activation of hedgehog signaling or the induction of EMT can attenuate even reverse obstruction-induced fibrosis (16). Thus, miRNAs concerning the regulation of hedgehog signaling or EMT may be potential targets for obstructive renal injury. A previous study by Ferretti et al (17) reported that miR-125b, miR-324-5p and miR-326 serve important roles in cerebellar neuronal progenitor and tumor cells through targeting the hedgehog signaling (Smo and Gli1). 
Other studies have identified that miR-125b and miR-326 regulate in several cells through suppressing the EMT process (18-20). In addition, miR-125b is critical for cardiac fibrosis, and miR-326 regulates profibrotic functions of tumor growth factor (TGF)- $\beta 1$ in pulmonary fibrosis $(20,21)$. Furthermore, miR-324-5p inhibits the induction of EMT and metastasis of cancer cells (22). Therefore, the authors hypothesized that miR-125b-5p, miR-324-5p and miR-326 may be correlated with obstructive renal injury, and as potential biomarkers for disease diagnosis or prognosis monitoring.

\section{Patients and methods}

Subjects. Consecutive peripheral blood specimens were collected between January 2015 and July 2015 from 76 controls and 91 patients with ureteral obstruction in the First Affiliated Hospital of Wenzhou Medical University (Wenzhou, China), and extra details about the participants are listed in Table I. All patients with ureteral obstruction were confirmed by B-scan ultrasonography, and the serum levels of creatinine ( $\mathrm{SCr}$ ), blood urea nitrogen (BUN), cholesterol (CHE), triglyceride (TG), high-density lipoprotein (HDL) and low density lipoprotein (LDL) in serum were examined by using an AU5800 automatic biochemistry analyzers (Beckman Coulter, Inc., Brea, CA, USA).

All subjects were informed about the contents of the study and gave their informed consent. This study was approved by the Ethics Committee of Wenzhou Medical University.

Sample preparation and miRNA extraction. Whole blood specimens were collected by venipuncture in EDTA K2-treated collection tubes and processed immediately or stored at $4^{\circ} \mathrm{C}$ overnight. A total of $0.7 \mathrm{ml}$ EDTA-treated whole blood and $2.1 \mathrm{ml}$ red blood cell lysis solution (R1010-500; Beijing Solarbio Science \& Technology Co., Ltd., Beijing, China) were pipetted into a tube and vortex gently for $20 \mathrm{sec}$. Then the tubes were put on ice for $15 \mathrm{~min}$, and gently homogenized mixing twice between blending. The white blood cells were collected by centrifugation at $450 \mathrm{xg}$ for $10 \mathrm{~min}$ at $4^{\circ} \mathrm{C}$. Red blood cell lysis solution $(1.4 \mathrm{ml})$ was added into the tube, and a suspension of white blood cells was made. The cells were collected by centrifugation at $450 \mathrm{x} g$ for $10 \mathrm{~min}$ at $4^{\circ} \mathrm{C}$. TRIzol reagent ( $1 \mathrm{ml}$; P/N: 15596-026; Ambion; Thermo Fisher Scientific, Inc., Waltham, MA, USA) were added into the collected cells and total RNA was extracted according to the manufacturer's instructions.

Reverse transcription-quantitative polymerase chain reaction $(R T-q P C R)$. RT was performed using the RevertAid First Strand cDNA Synthesis kit (Thermo Fisher Scientific, Inc.). The $10 \mu \mathrm{l}$ RT reaction mixture contained the following: $1 \mu \mathrm{l}$ treated RNA (0.1 ng-5 mg), $1 \mu$ l RT stem-loop primer $(5 \mu \mathrm{M})$ and $1 \mu \mathrm{l}$ U6 RT primer $(5 \mu \mathrm{M}), 1 \mu \mathrm{l}$ dNTP Mix (10 mM), $2 \mu \mathrm{l}$ $5 \mathrm{X}$ reaction buffer (cat no. K1622), $0.5 \mu 1$ Ribolock RNase inhibitor $(20 \mathrm{U} / \mathrm{ml}$; all Thermo Fisher Scientific, Inc.), and $0.5 \mu \mathrm{l}$ RevertAid M-MuLV reverse transcriptase $(200 \mathrm{U} / \mathrm{ml}$; Thermo Fisher Scientific, Inc.). The mixture was incubated at $25^{\circ} \mathrm{C}$ for $5 \mathrm{~min}$, and then incubation was continued at $42^{\circ} \mathrm{C}$ for $60 \mathrm{~min}$. The reaction was inactivated by heating at $70^{\circ} \mathrm{C}$ for $5 \mathrm{~min}$. The $\mathrm{RT}$ reaction was performed in triplicate to remove RT outliers.
RT-qPCR was performed using SYBR-Green fluorescence quantitative PCR reagent kit (cat. no. QPS-201T; Toyobo Co., Ltd., Osaka, Japan) on a 7500 real-time quantitative PCR instrument (Applied Biosystems; Thermo Fisher Scientific, Inc.), and each sample was analyzed in triplicate. The $10 \mu \mathrm{lPCR}$ volume included $1 \mu 1 \mathrm{RT}$ product, $5 \mu \mathrm{l} \mathrm{SYBR}$-Green real-time PCR Master Mix (Roche Diagnostics, Basel, Switzerland), and $1 \mu \mathrm{l}$ primer $(1 \mu \mathrm{M})$. The reactions were incubated at $95^{\circ} \mathrm{C}$ for $3 \mathrm{~min}$, followed by 40 cycles of $95^{\circ} \mathrm{C}$ for $5 \mathrm{sec}, 58^{\circ} \mathrm{C}$ for $35 \mathrm{sec}$. The expression levels of miRNAs (miR-125b-5p, miR-324 and miR-326) were analyzed by calculating the equation $2^{-\Delta \Delta C q}$ and normalized to U6 expression $(23,24)$. All primers are presented in Table II.

Statistical analysis. Statistical analysis was performed using SPSS software (version 19.0; IBM SPSS, Armonk, NY, USA). All data were presented as mean \pm standard deviation with normal distribution, and median (lower and upper quartiles) for the others. A binary logistic regression analysis was conducted to adjust the influence of age and gender. Data were compared by the Mann-Whitney U test and Spearman's rank order correlations as appropriate. $\mathrm{P}<0.05$ was considered to indicated a statistically significant difference. All probabilities were two-tailed.

\section{Results}

Risk assessment of obstructive renal injury. The demographic and baseline clinical data of the study subjects are summarized in Table I. The serum levels of $\mathrm{SCr}(\mathrm{P}<0.001)$ and BUN $(\mathrm{P}=0.01)$ in patients with ureteral obstruction were significantly higher than those in controls, indicating that ureteral obstruction induced renal injury and function decline, though the extent of renal injury did not reach the diagnosis standard of nephropathy $(97 \mu \mathrm{mol} / \mathrm{l})$. In addition, the serum levels of HDL in patients with ureteral obstruction are significantly lower than those in controls $(\mathrm{P}<0.001)$, suggesting that patients with ureteral obstruction have a higher risk of hyperlipidemia.

Levels of miRNAs in controls and patients with obstructive renal injury. As presented in Table III, there were strong internal correlations in levels among these three miRNAs $(\mathrm{P}<0.001)$. Data were compared by Spearman's rank order correlations.

The expression levels of miR-125b-5p, miR-324-5p and miR-326 are summarized in patients with ureteral obstruction and controls (Fig. 1). The results demonstrated that the expression of miR-324-5p [1.003 (0.391-2.279) vs. 0.934 (0.579-1.539), $\mathrm{P}=0.300]$ and miR-326 [0.840 (0.180-2.020) vs. $0.949(0.507-1.702), \mathrm{P}=0.050]$ did not suggest any significant difference (Figs. 1A and B). However, the expression of miR-125b-5p [0.755 (0.210-2.110) vs. 0.960 (0.390-1.770), $\mathrm{P}=0.002$ ] in patients with ureteral obstruction was significantly lower than those in controls, indicating that miR-125b-5p may be potential protective biomarkers in patients with obstructive renal injury (Fig. 1C). Interestingly, the authors identified that the protective effect of miR-125b-5p has a significant correlation with gender and age. In patients with ureteral obstruction, the levels of miR-125b-5p in male were lower than those in female [0.965 (0.833-1.097) vs. 1.222 (1.055-1.389), $\mathrm{P}=0.0171$; 
Table I. Demographic and baseline clinical data.

\begin{tabular}{|c|c|c|c|c|}
\hline Characteristics & Control $(n=76)$ & Patients with ureteral obstruction $(n=91)$ & Odds ratio $(95 \% \mathrm{CI})$ & P-value \\
\hline \multicolumn{5}{|l|}{ Gender } \\
\hline Male & $41(53.9 \%)$ & $53(58.2 \%)$ & $1.19(0.64-2.20)$ & $0.577^{\mathrm{b}}$ \\
\hline Female & $35(46.1 \%)$ & $38(41.8 \%)$ & & \\
\hline Age (year) & $42.20 \pm 12.79$ & $53.75 \pm 12.40$ & & $<0.001^{\mathrm{a}}$ \\
\hline$<50$ & $12(14.8 \%)$ & $29(31.9 \%)$ & $2.50(1.17-5.32)$ & $0.016^{\mathrm{b}}$ \\
\hline$\geq 50$ & $64(84.2 \%)$ & $62(68.1 \%)$ & & \\
\hline \multicolumn{5}{|c|}{ Urinary tract obstruction } \\
\hline Unilateral (left) & 0 & $49(53.8 \%)$ & & \\
\hline Unilateral (right) & 0 & $31(34.1 \%)$ & & \\
\hline Bilateral & 0 & $9(9.9 \%)$ & & \\
\hline \multicolumn{5}{|l|}{ Renal function } \\
\hline $\mathrm{SCr}(\mu \mathrm{mol} / \mathrm{l})$ & $64.76 \pm 12.55$ & $84.32 \pm 34.88$ & & $<0.001^{\mathrm{a}}$ \\
\hline BUN (mmol/l) & $5.09 \pm 1.16$ & $7.03 \pm 8.01$ & & $0.01^{\mathrm{a}}$ \\
\hline \multicolumn{5}{|l|}{ Serum lipid levels } \\
\hline CHE (mmol/l) & $5.21 \pm 1.00$ & $4.87 \pm 0.96$ & & $0.04^{\mathrm{a}}$ \\
\hline TG (mmol/l) & $1.90 \pm 1.43$ & $1.79 \pm 1.14$ & & $0.93^{\mathrm{a}}$ \\
\hline $\mathrm{HDL}(\mathrm{mmol} / \mathrm{l})$ & $1.32 \pm 0.50$ & $1.08 \pm 0.31$ & & $<0.001^{\mathrm{a}}$ \\
\hline $\mathrm{LDL}(\mathrm{mmol} / \mathrm{l})$ & $3.10 \pm 0.74$ & $2.87 \pm 0.80$ & & $0.12^{\mathrm{a}}$ \\
\hline
\end{tabular}

${ }^{a}$ Data are expressed as mean \pm standard deviation. P-values are calculated using unpaired t-test. ${ }^{b}$ Based on Chi-squared test. SCr, serum creatinine; BUN, blood urea nitrogen; CHE, cholesterol; TG, triglyceride; HDL, high-density lipoprotein; LDL, low-density lipoprotein; CI, confidence interval.

Table II. The primers of RT-quantitative polymerase chain reaction.

\begin{tabular}{llcc}
\hline Primer & RT stem-loop primer sequence (5'-3') & Forward primer sequence (5'-3') & Reverse primer sequence (5'-3') \\
\hline miR-125b-5p & GAAAGAAGGCGAGGAGCAGATCG & CGCTCCCTGA & CGAGGAAGAA \\
& AGGAAGAAGACGGAAGAATGTGC & GACCCTAACT & GACGGAAGAAT \\
GTCTCGCCTTCTTTCTCACAAGT & & CGAGGAAGAA \\
& GAAAGAAGGCGAGGAGCAGATCG & CGCATCCCC & GACGGAAGAAT \\
miR-326 & AGGAAGAAGACGGAAGAATGT & TAGGGCAT & CGAGGAAGAA \\
& GAGTCTCGCCTTCTTTCACACCAAT & & GACGGAAGAAT \\
U6 & AGGAAGAAGACGGAAGAATGTGCG & GCCCTTCC & CGCTTCACGAA \\
& TCTCGCCTTCTTCCTGGAGGA & & TTTGCGTGTCAT
\end{tabular}

miR, microRNA; RT, reverse transcription.

Fig. 2A]. Moreover, the levels of miR-125b-5p in the older patients ( $\geq 50$ years) were lower than those in the younger patients $[<50$ years, $0.966(0.801-1.131)$ vs. 1.359 (1.066-1.652), $\mathrm{P}=0.0142$; Fig. 2B]. Thus, higher levels of miR-125b-5p in younger and female patients may be an important protective biomarker against obstructive renal injury.

Association between miR-125b-5p levels and renal function. Given that miR-125b-5p can exert its protective effects on obstructive renal injury, the authors investigated whether the effects of miR-125b-5p correlated with renal function. As demonstrated in Fig. 3, the levels of miR-125b-5p ( $r=-0.175$, $\mathrm{P}=0.038$ ) in patients with ureteral obstruction were significantly negatively correlated to the serum levels of SCr, though not correlated to the levels of BUN ( $P>0.05)$. These results confirmed again to some extent that the protective roles of miR-125b-5p in obstructive renal injury may be associated with downregulated SCr levels. There were no significant 
Table III. Internal correlation among the miRNAs.

\begin{tabular}{lcc}
\hline Level & miR-125b-5p & miR-324-5p \\
\hline miR-324-5p & & - \\
r & 0.764 & - \\
P & $<0.001$ & \\
miR-326 & & 0.842 \\
r & 0.858 & $<0.001$ \\
P & $<0.001$ & \\
\hline
\end{tabular}

miR/miRNA, microRNA.

Table IV. Internal correlation between miRNA levels and serum lipid levels.

\begin{tabular}{lcccc}
\hline Level & CHE & TG & HDL & LDL \\
\hline miR-125b-5p & & & & \\
r & -0.014 & 0.059 & -0.027 & -0.022 \\
P & 0.869 & 0.485 & 0.748 & 0.794 \\
miR-324-5p & & & & \\
r & -0.112 & -0.045 & -0.152 & -0.046 \\
P & 0.182 & 0.595 & 0.067 & 0.580 \\
miR-326 & & & & \\
r & -0.057 & -0.042 & -0.026 & 0.001 \\
P & 0.496 & 0.614 & 0.750 & 0.988 \\
\hline
\end{tabular}

CHE, cholesterol; TG, triglyceride; HDL, high-density lipoprotein; LDL, low-density lipoprotein; miRNA/miR, microRNA.

internal correlation between miRNA levels and serum lipid levels ( $\mathrm{P}>0.05$; Table IV).

\section{Discussion}

Obstruction of the ureter resulted in renal dysfunction with marked increases in BUN and SCr $(2,25)$. In the current study, the authors demonstrated that the levels of $\mathrm{SCr}$ in patients with ureteral obstruction were significant higher than those in controls. Upregulated levels of SCr following ureteral obstruction indicated that the kidney was injured, but the extent of injury did not reach the diagnosis standard of nephropathy, and thereby did not cause marked renal dysfunction. A previous study has indicated that HDL can reduce renal ischemia/reperfusion injury and mortality via modulation of the expression of adhesion molecules and pro-inflammatory enzymes (26). HDL levels in patients with ureteral obstruction were significantly lower $(\mathrm{P}<0.001)$, when compared with control samples, identifying the protective role of HDL in obstructive renal injury.

A number of studies have demonstrated that miRNAs were involved in the development and progression of obstructive renal injury and fibrosis $(27,28)$. Smad3-mediated upregulation of miR-21 promotes fibrosis in established obstructive renal injury (27). TGF- $\beta / \mathrm{Smad} 3$ signaling promotes renal
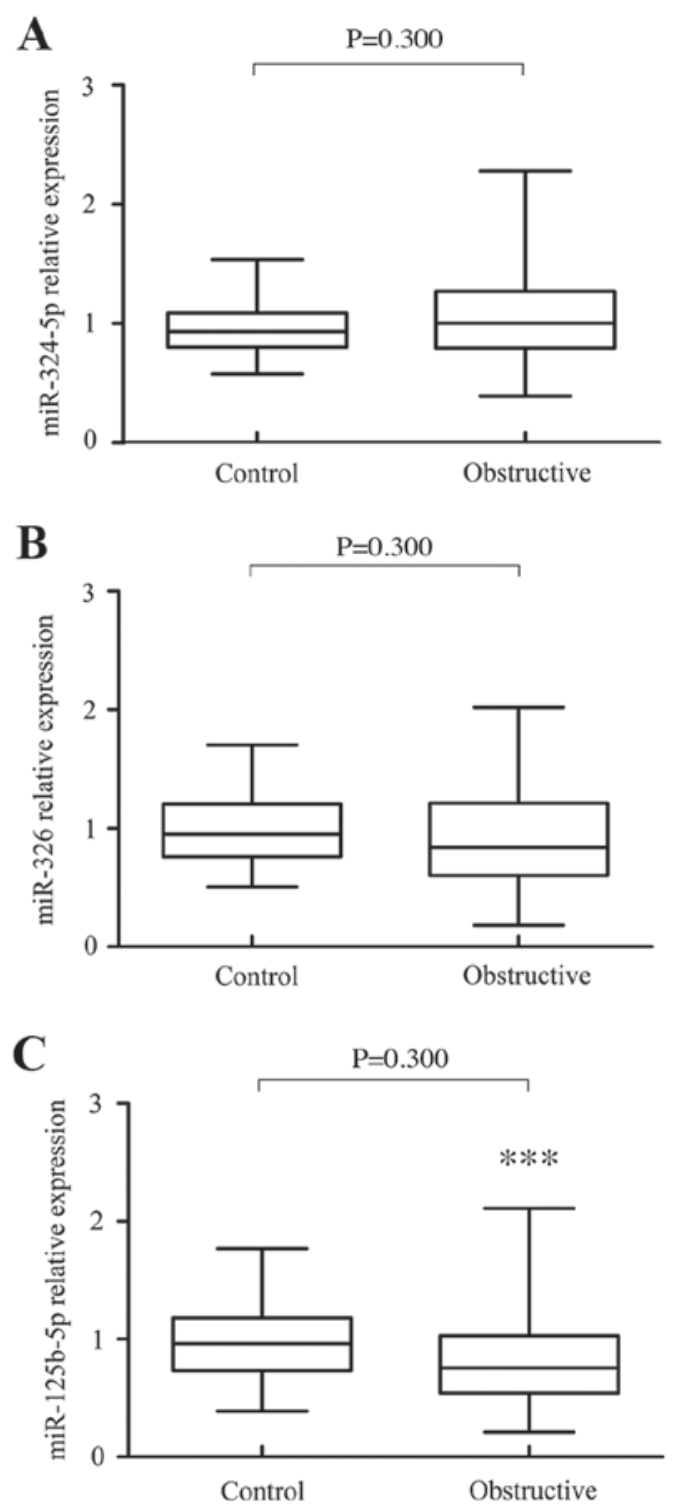

Figure 1. miRNA levels determined by reverse transcription-quantitative polymerase chain reaction in controls and patients with ureteral obstruction. (A) There was no significant difference in miR-324-5p levels between patients with ureteral obstruction [1.003 (0.391-2.279)] and controls [0.934 (0.579-1.539), $\mathrm{P}=0.300]$. (B) There was no significant difference in miR-326 levels between in patients with ureteral obstruction $[0.840(0.180-2.020)]$ and controls [0.949 (0.507-1.702), $\mathrm{P}=0.050]$. (C) The expression of miR-125b-5p in patients with ureteral obstruction $[0.755(0.210-2.110)]$ was significantly lower than those in controls [0.960 (0.390-1.770), $\mathrm{P}=0.002] .{ }^{* * *} \mathrm{P}<0.001$ vs. control. miRNA, microRNA.

fibrosis by inhibiting miR-29 (28). miR-192 mediates TGF-beta/Smad3-driven renal fibrosis (29). In the current study, levels of miR-125b-5p in patients with ureteral obstruction were significantly lower than those in controls, though miR-324-5p levels did not present any significant difference. Moreover, compared with the younger or female patients, the levels of miR-125b-5p in male or elder patients were lower. Further examination revealed that miR-125b-5p levels were negatively correlated with SCr levels, suggesting that miR-125b-5p may be a protective biomarker for obstructive renal injury. However, the levels of miR-125b-5p were not associated with serum lipid levels, indicating that the effects of 
A

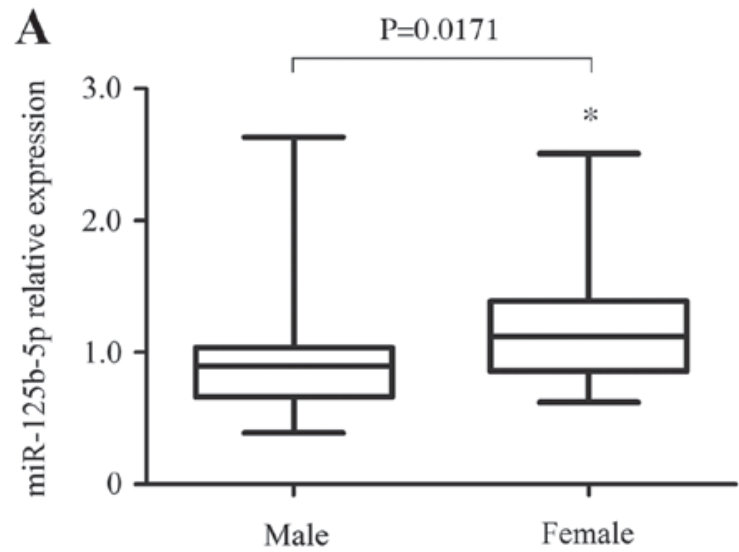

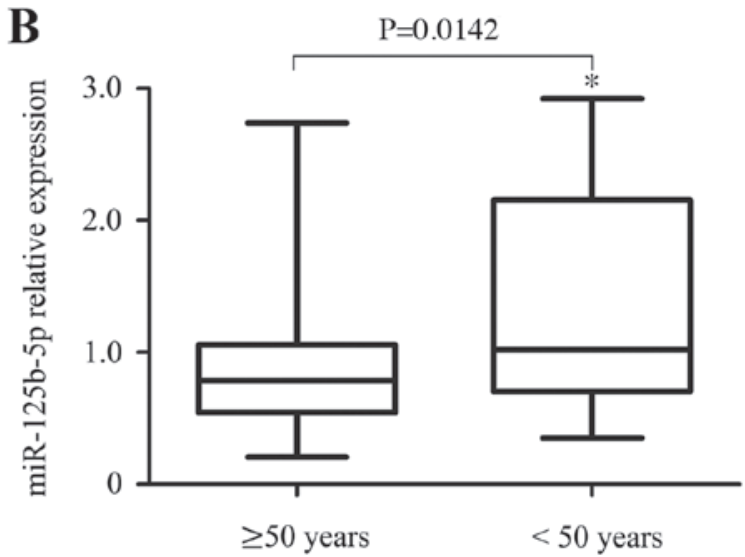

Figure 2. Comparison of miR-125b-5p levels in patients with ureteral obstruction according to different gender and age. (A) The levels of miR-125b-5p in male patients with ureteral obstruction [0.965 (0.833-1.097)] were lower than those in females [1.222 (1.055-1.389), $\mathrm{P}=0.0171]$. (B) The levels of miR-125b-5p in older patients with ureteral obstruction $[\geq 50$ years, $0.966(0.801-1.131)]$ were lower than those in the younger patients $[<50$ years, $1.359(1.066-1.652)$, $\mathrm{P}=0.0142] .{ }^{*} \mathrm{P}<0.05$ vs. control. miR, microRNA.
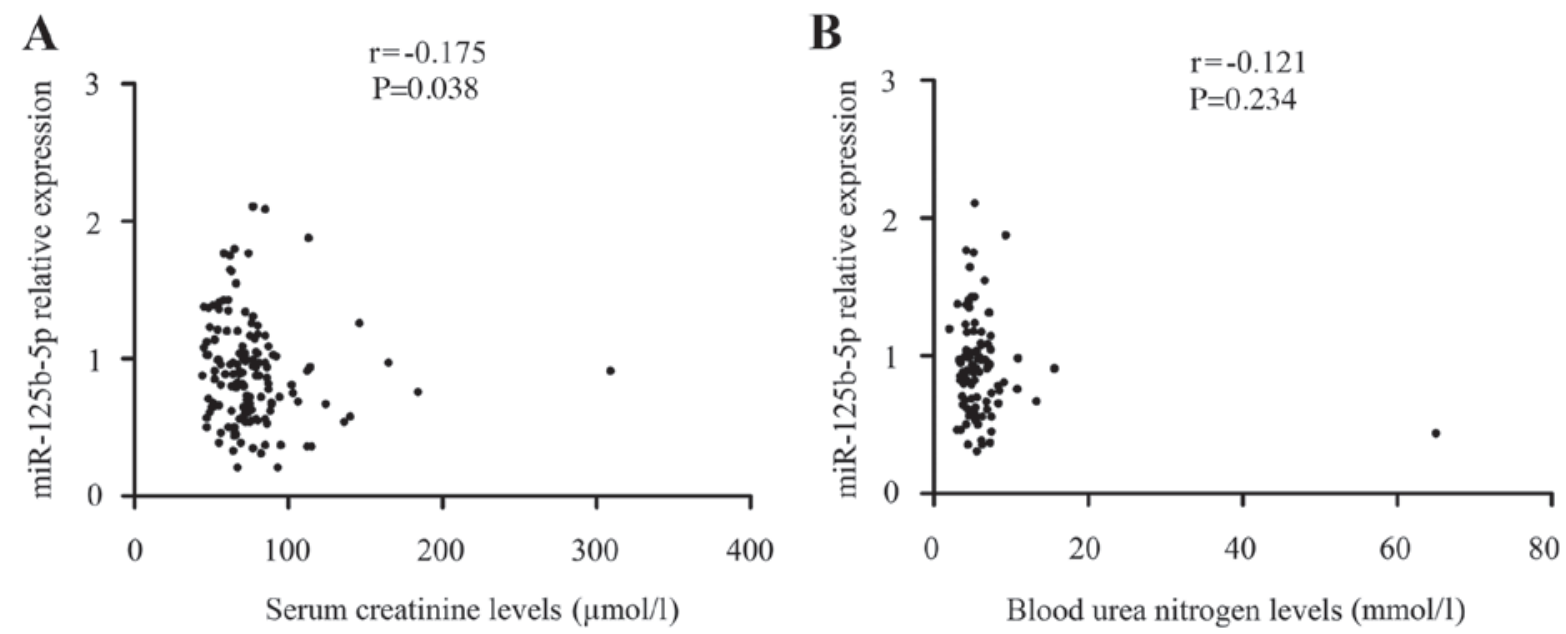

Figure 3. Relationship between miR-125b-5p levels and renal function. (A) There is a clear correlation in patients with ureteral obstruction between the levels of miR-125b-5p and the serum levels of SCr $(\mathrm{r}=-0.175, \mathrm{P}=0.038)$. (B) The levels of miR-125b-5p in patients with ureteral obstruction were not correlated to the serum levels of BUN ( $r=-0.121, \mathrm{P}=0.234)$. miR, microRNA.

miR-125b-5p and serum lipid on renal injury are independent of each other.

Currently, the roles and functions of miR-125b-5p in obstructive renal injury remain unknown. A previous study of the authors indicated that injury-induced activation of the hedgehog signaling pathway stimulated TGF- $\beta 1$ expression and promoted the induction of EMT, resulted in excessive ECM component synthesis and deposition in the cortical interstitium of kidneys (16). In vivo and in vitro, the activity of hedgehog signaling may be regulated by miR-125b, which serves an important role in cell proliferation and differentiation and tissue regeneration $(17,30)$. miR-125b exerts its inhibitory effects on EMT and EMT-associated traits in tumorigenesis by antagonizing TGF- $\beta 1 / \mathrm{Smad}$ signaling pathway (19). Taken together, these findings provide some suggestive evidence of the protective biomarkers of miR-125b-5p for obstructive renal injury. Further studies should be performed to investigate the functions of these two miRNAs involved in the regulation of the hedgehog and TGF- $\beta 1 /$ Smad signaling pathway in various experimental models.
However, it should be noted that there are some other limitations in the present study. The sample size was too small to evaluate the stage of renal injury in patients with ureteral obstruction due to the lack of pathological evidence. Additionally, these results revealed that there were strong correlations in levels among three miRNAs, but we did not know how to interact with each other. Furthermore, whether these miRNAs can be used as biomarkers in early diagnosis and detecting disease development, and whether to have high sensitivity and specificity should be further studied.

In conclusion, these findings indicated that the expression levels of miR-125b-5p and miR-326 were decreased in patients with ureteral obstruction, and correlated with the loss of renal function. Thus, miR-125b-5p may be a potential protective biomarker for obstructive renal injury.

\section{Acknowledgements}

The current study was sponsored by the Natural Science Foundation of Zhejiang Province (grant no. LY17H050005) and 
the Wenzhou Municipal Science and Technology Plan Project (grant no. Y20150037). The project was also supported by the Natural Science Foundation of China (grant no. 81572087).

\section{References}

1. Dikmen B, Yagmurdur H, Akgul T, Astarci M, Ustun H and Germiyanoglu C: Preventive effects of propofol and ketamine on renal injury in unilateral ureteral obstruction. J Anesth 24: 73-80, 2010.

2. Ucero AC, Benito-Martin A, Izquierdo MC, Sanchez-Niño MD, Sanz AB, Ramos AM, Berzal S, Ruiz-Ortega M, Egido J and Ortiz A: Unilateral ureteral obstruction: Beyond obstruction. Int Urol Nephrol 46: 765-776, 2014.

3. Kinn AC and Bohman SO: Renal structural and functional changes after unilateral ureteral obstruction in rabbits. Scand J Urol Nephrol 17: 223-234, 1983.

4. Klahr S: New insights into the consequences and mechanisms of renal impairment in obstructive nephropathy. Am J Kidney Dis 18: 689-699, 1991.

5. Lee RC, Feinbaum RL and Ambros V: The C. elegans heterochronic gene lin-4 encodes small RNAs with antisense complementarity to lin-14. Cell 75: 843-854, 1993.

6. Glowacki F, Savary G, Gnemmi V, Buob D, Van der Hauwaert C, Lo-Guidice JM, Bouyé S, Hazzan M, Pottier N, Perrais M, et al: Increased circulating miR-21 levels are associated with kidney fibrosis. PLoS One 8: e58014, 2013.

7. Sayed D and Abdellatif M: MicroRNAs in development and disease. Physiol Rev 91: 827-887, 2011.

8. Wang G, Kwan BC, Lai FM, Chow KM, Li PK and Szeto CC: Urinary miR-21, miR-29, and miR-93: Novel biomarkers of fibrosis. Am J Nephrol 36: 412-418, 2012.

9. Aguado-Fraile E, Ramos E, Conde E, Rodríguez M, Martín-Gómez L, Lietor A, Candela Á, Ponte B, Liaño F and García-Bermejo ML: A Pilot Study Identifying a Set of microRNAs As Precise Diagnostic Biomarkers of Acute Kidney Injury. PLoS One 10: e0127175, 2015.

10. Wang B, Komers R, Carew R, Winbanks CE, Xu B, Herman-Edelstein M, Koh P, Thomas M, Jandeleit-Dahm K, Gregorevic P, et al: Suppression of microRNA-29 expression by TGF- $\beta 1$ promotes collagen expression and renal fibrosis. J Am Soc Nephrol 23: 252-265, 2012.

11. Aguado-Fraile E, Ramos E, Sáenz-Morales D, Conde E, Blanco-Sánchez I, Stamatakis K, del Peso L, Cuppen E, Brüne B and Bermejo ML: miR-127 protects proximal tubule cells against ischemia/reperfusion: Identification of kinesin family member 3B as miR-127 target. PLoS One 7: e44305, 2012.

12. Fan PC, Chen CC, Chen YC, Chang YS and Chu PH: MicroRNAs in acute kidney injury. Hum Genomics 10: 29, 2016.

13. Liu Z, Wang S, Mi QS and Dong Z: MicroRNAs in Pathogenesis of Acute Kidney Injury. Nephron 134: 149-153, 2016.

14. Bai Y, Lu H, Lin C, Xu Y, Hu D, Liang Y, Hong W and Chen B: Sonic hedgehog-mediated epithelial-mesenchymal transition in renal tubulointerstitial fibrosis. Int J Mol Med 37: 1317-1327, 2016.

15. Hu L, Lin X, Lu H, Chen B and Bai Y: An overview of hedgehog signaling in fibrosis. Mol Pharmacol 87: 174-182, 2015.
16. Bai Y, Lu H, Wu C, Liang Y, Wang S, Lin C, Chen B and Xia P: Resveratrol inhibits epithelial-mesenchymal transition and renal fibrosis by antagonizing the hedgehog signaling pathway. Biochem Pharmacol 92: 484-493, 2014.

17. Ferretti E, De Smaele E, Miele E, Laneve P, Po A, Pelloni M, Paganelli A, Di Marcotullio L, Caffarelli E, Screpanti I, et al: Concerted microRNA control of Hedgehog signalling in cerebellar neuronal progenitor and tumour cells. EMBO J 27: 2616-2627, 2008

18. Zhang J, Na S, Liu C, Pan S, Cai J and Qiu J: MicroRNA-125b suppresses the epithelial-mesenchymal transition and cell invasion by targeting ITGA9 in melanoma. Tumour Biol 37: 5941-5949, 2016.

19. Zhou JN, Zeng Q, Wang HY, Zhang B, Li ST, Nan X, Cao N, Fu CJ, Yan XL, Jia YL, et al: MicroRNA-125b attenuates epithelial-mesenchymal transitions and targets stem-like liver cancer cells through small mothers against decapentaplegic 2 and 4. Hepatology 62: 801-815, 2015.

20. Das S, Kumar M, Negi V, Pattnaik B, Prakash YS, Agrawal A and Ghosh B: MicroRNA-326 regulates profibrotic functions of transforming growth factor- $\beta$ in pulmonary fibrosis. Am J Respir Cell Mol Biol 50: 882-892, 2014.

21. Nagpal V, Rai R,Place AT, Murphy SB, Verma SK, Ghosh AK and Vaughan DE: miR-125b Is Critical for Fibroblast-to-Myofibroblast Transition and Cardiac Fibrosis. Circulation 133: 291-301, 2016.

22. Song L, Liu D, Zhao Y, He J, Kang H, Dai Z, Wang X, Zhang S and Zan Y: Sinomenine inhibits breast cancer cell invasion and migration by suppressing $\mathrm{NF}-\kappa \mathrm{B}$ activation mediated by IL-4/miR-324-5p/CUEDC2 axis. Biochem Biophys Res Commun 464: 705-710, 2015.

23. Bustin SA, Benes V, Garson JA, Hellemans J, Huggett J, Kubista M, Mueller R, Nolan T, Pfaffl MW, Shipley GL, et al: The MIQE guidelines: Minimum information for publication of quantitative real-time PCR experiments. Clin Chem 55: 611-622, 2009.

24. Livak KJ and Schmittgen TD: Analysis of relative gene expression data using real-time quantitative PCR and the 2(-Delta Delta C(T)) Method. Methods 25: 402-408, 2001.

25. Butler J, Chirovsky D, Phatak H, McNeill A and Cody R: Renal function, health outcomes, and resource utilization in acute heart failure: A systematic review. Circ Heart Fail 3: 726-745, 2010.

26. Thiemermann C, Patel NS, Kvale EO, Cockerill GW, Brown PA, Stewart KN, Cuzzocrea S, Britti D, Mota-Filipe $\mathrm{H}$ and Chatterjee PK: High density lipoprotein (HDL) reduces renal ischemia/reperfusion injury. J Am Soc Nephrol 14: 1833-1843, 2003.

27. Zhong X, Chung AC, Chen HY, Meng XM and Lan HY: Smad3-mediated upregulation of miR-21 promotes renal fibrosis. J Am Soc Nephrol 22: 1668-1681, 2011.

28. Qin W, Chung AC, Huang XR, Meng XM, Hui DS, Yu CM, Sung JJ and Lan HY: TGF- $\beta /$ Smad3 signaling promotes renal fibrosis by inhibiting miR-29. J Am Soc Nephrol 22: 1462-1474, 2011.

29. Chung AC, Huang XR, Meng X and Lan HY: miR-192 mediates TGF-beta/Smad3-driven renal fibrosis. J Am Soc Nephrol 21: $1317-1325,2010$

30. Hyun J, Wang S, Kim J, Kim GJ and Jung Y: MicroRNA125b-mediated Hedgehog signaling influences liver regeneration by chorionic plate-derived mesenchymal stem cells. Sci Rep 5: 14135, 2015. 